\title{
Pseudarthrosen im Mund-Kiefer-Gesichts-Bereich
}

\author{
Andreas Neff, Jonas Jung
}

- Tab. 1 ICD-10-Diagnosen im Zusammenhang mit Pseudarthrosen.

\begin{tabular}{|c|c|}
\hline $\begin{array}{l}\text { ICD-10-Diagnose- } \\
\text { bezeichnungen }\end{array}$ & Beschreibung \\
\hline S02.0-S02.9 & Fraktur des Schädels und Gesichtsschädelknochens \\
\hline $\begin{array}{l}\text { C41.01, C41.02, } \\
\text { C41.1 }\end{array}$ & $\begin{array}{l}\text { bösartige Neubildung: Knochen im Gesicht und } \\
\text { Hirnschädel }\end{array}$ \\
\hline M84.08 & Veränderung der Knochenkontinuität \\
\hline K07.0-K0.9 & dentofaziale Anomalie \\
\hline K10.0, K10.2, K10.8 & sonstige Krankheiten des Kiefers \\
\hline T90.2 & $\begin{array}{l}\text { Folgen einer Fraktur des Schädels und Gesichts- } \\
\text { schädelknochens }\end{array}$ \\
\hline
\end{tabular}
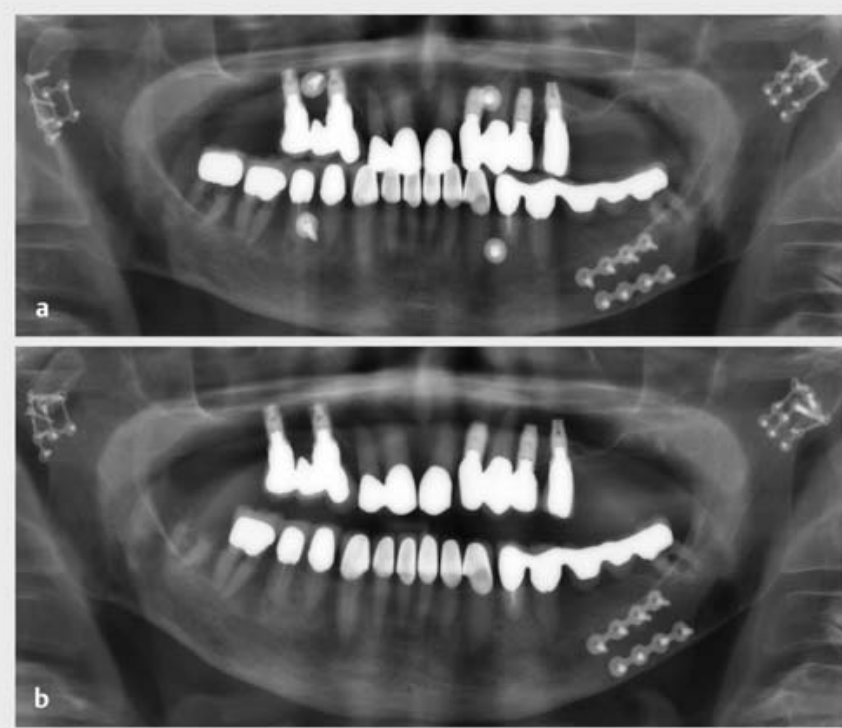

- Abb. 1 a Orthopantomogramm: Kontrolle nach operativer Versorgung einer trifokalen Unterkieferfraktur mit bilateraler Gelenkhalsfraktur [20] bei einer 88-jährigen Patientin. Aufgrund von Osteoporose konnten nur Platten des 1,5-mm-Systems eingesetzt werden (üblicherweise sonst 2,0mm-Systeme). b Orthopantomogramm: Verlaufskontrolle zeigt eine sekundäre Dislokation im Bereich des rechten Gelenkhalses mit Osteolysezone im Bereich des gelockerten Osteosynthesematerials (mit Revisionsindikation).

\section{Einleitung}

Die moderne funktionsstabile Osteosynthese basiert auf den Prinzipien von Champy und Michelet [1], die die Osteosynthese im Bereich der Mund-, Kiefer- und Gesichtschirurgie (MKG-Chirurgie) grundlegend revolutioniert haben. Während im Zeitalter der Drahtnahtosteosynthese Pseudarthrosen bis in die 1980er-Jahre eine häufige Komplikation darstellten [2-4], ist es heute möglich, eine effiziente Versorgung unter den Primärzielen einer anatomisch und ästhetisch korrekten Rekonstruktion sowie unter Wiederherstellung der Kaufunktion durchzuführen. Pseudarthrosen, die eine Revision nach Osteosynthese bzw. Rekonstruktion erforderlich machen, treten daher in Standardsituationen heute deutlich seltener auf. Allerdings finden sich weiterhin Pseudarthrosen in verschiedensten Bereichen der MKG-Chirurgie. Zu nennen sind hier die Traumatologie (z.B. Frakturen mit Majorfragmentation $[5,6])$, die orthognathe Chirurgie und insbesondere die rekonstruktive Chirurgie mit den aufwendigen mikrovaskulären Rekonstruktionen (z.B. Fibula-, Skapula- und Beckenkammtransplantate zur subtotalen Unterkieferrekonstruktion). > Tab. 1 gibt einen Überblick über die im Zusammenhang stehenden ICD-10-Diagnosen.

\section{Pseudarthrosen - Definition und Grundsätze der Therapie}

Unter einer biologischen Betrachtungsweise ist es wichtig, im klinischen Sprachgebrauch zwischen einer verzögerten Bruchheilung und einer Pseudarthrose zu unterscheiden. Eine Pseudarthrose liegt demnach erst dann vor, wenn sich zwischen den Fragmenten ein hochdifferenziertes, faserreiches Gewebe ausgebildet hat. Haben sich an den Fragmentstümpfen kortikale Deckschichten im Sinne einer zarten Lamina dura [4] ausgebildet, ist die Osteogenese abgeschlossen, im Bereich des ehemaligen Frakturspalts liegt keine osteogene Potenz mehr vor [7]. Grundsätzlich gilt, dass eine Pseudarthrose dann entsteht, wenn die Bruchstellen durch die Osteosynthese nicht ausreichend stabilisiert wurden, Weichgewebe interponiert sind, eine sekundäre Dislokation der Fragmente ( $\triangleright$ Abb. $\mathbf{1}$ ) aufgetreten ist und/oder ein größerer Frakturspalt oder Hartgewebsdefekt nicht ausreichend knöchern überbrückt werden kann. In der Folge kann 

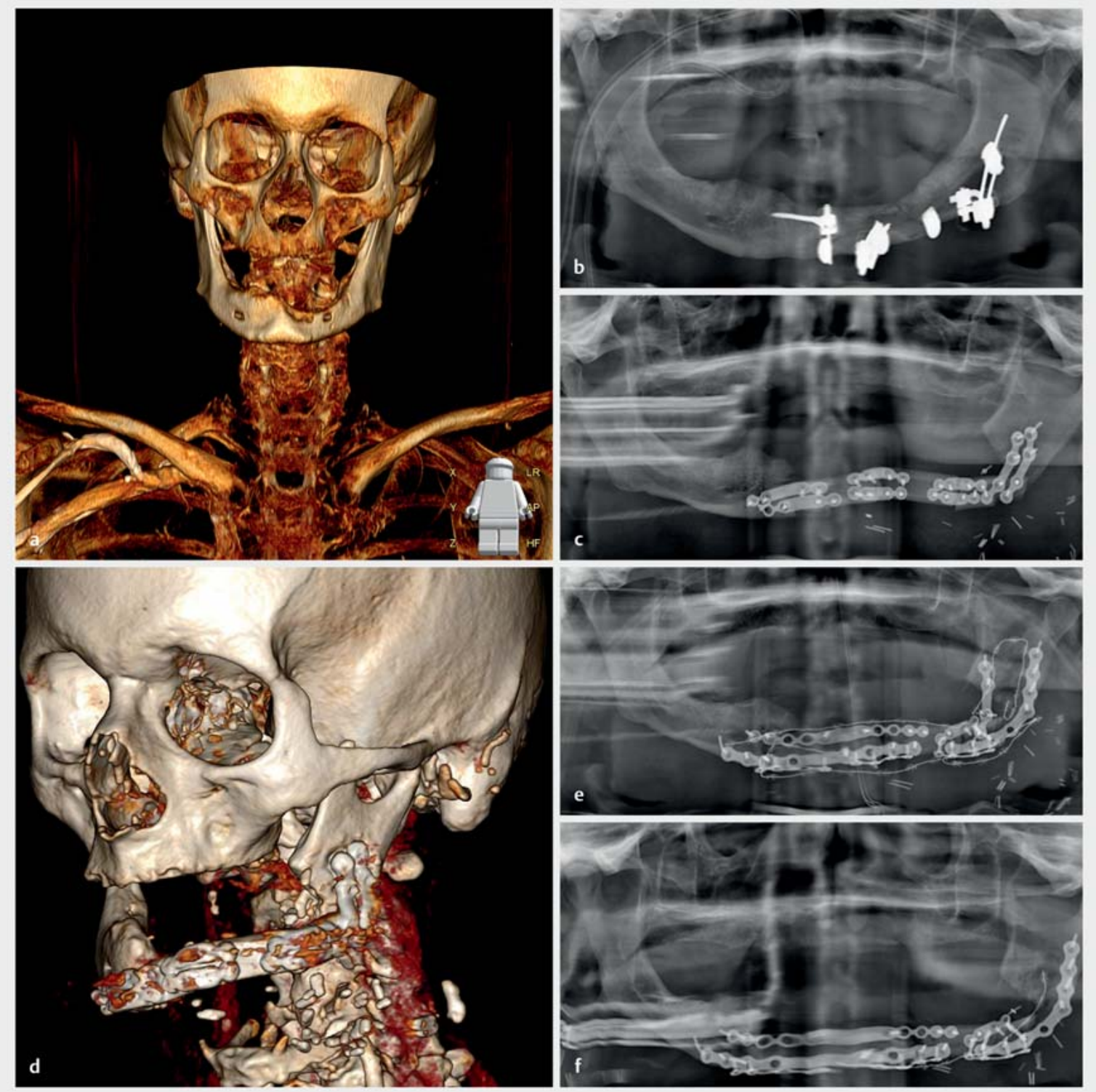

- Abb. 2 a 3-D-CT: Ausgangsbefund mit Unterkieferdefekt nach wiederholten Sequestrotomien und modellierenden Osteotomien bei Osteoradionekrose. b Orthopantomogramm: pathologische Fraktur im linken Unterkieferkorpus bei über einen intraoralen Weichteildefekt infizierter Osteoradionekrose, mit Fixateur externe temporär stabilisiert. c Orthopantomogramm: Unterkieferrekonstruktion mit 3-fach segmentierter Fibula, Osteosynthese mit jeweils doppelten Locking-Platten (System 2,0 mm) im Kinn- und Korpusbereich, 2,3-mm-Locking-Platten für den Kieferwinkel, die intraorale Defektdeckung erfolgte mit Fibulahautinsel. d 3-D-CT: Unterkieferrekonstruktion mit segmentiertem Fibulatransplantat bei LC-Defekt (LC: lateraler Defekt der Mandibula bei Erhalt des Kiefergeenkfortsatzes kombiniert mit Defekt der gesamten Kinnregion, vergleiche $>$ Tab. 3 [41]). e Orthopantomogramm: Einlage einer Septopalkette und Plattenwechsel nach Infektion des Transplantats (Keime des Mundhöhlenspektrums), klinisch besteht eine Fistelung nach extraoral, jedoch keine Perforation nach intraoral. f Orthopantomogramm: Kontrollaufnahme nach erneutem Plattenwechsel bei persistierender Fistelung. Beachte die Resorptionen im Kieferwinkelbereich und die jetzt breiteren Klaffungen zwischen den Segmenten im Sinne einer manifesten intersegmentalen Pseudarthrosenbildung.

der betroffene Kieferabschnitt eine Hypermobilität aufweisen und/oder ein Falschgelenk entstehen. Das Ausmaß der dabei auftretenden Wackel- oder Fehlbewegungen ist abhängig von der Breite und Straffheit der bindegewebigen Brücke zwischen den nicht knöchern vereinigten Fragmenten. Pseudarthrosen treten an der biomechanisch stark belasteten Mandibula deutlich häufiger auf als an der Maxilla, bei Letzterer dann ggf. in der LeFort-I-Ebene. Für das Auftreten einer Pseudarthrose sind außerdem Komorbiditäten wie Diabetes mellitus, arterielle Verschlusskrankheiten, systemische Infekte oder lokale Infektionen von Bedeutung, die besonders bei Patienten nach Strahlentherapie zum Tragen kommen ( $\bullet$ Abb. 2). Die Diagnose einer Pseudarthrose wird gestellt, wenn 
- Tab. 2 Kategorien der Pseudarthrose, Klinik und Therapieprinzipien.

\begin{tabular}{|l|l|l|}
\hline Form & Klinik & Therapie \\
\hline hypertroph/vital & vermehrte Knochen- und Knorpelneubildung & Resektion und Stabilisierung (Osteosynthese) \\
\hline atroph/avital & $\begin{array}{l}\text { Nonunion } \\
\text { z. B. bei gestörter Trophik }\end{array}$ & $\begin{array}{l}\text { Débridement und Osteosynthesewechsel } \\
\text { (Kortiko-)Spongiosatransfer }\end{array}$ \\
\hline entzündlich & Infektion & $\begin{array}{l}\text { Débridement und Osteosynthesewechsel } \\
\text { ggf. (Kortiko-)Spongiosatransfer }\end{array}$ \\
\hline
\end{tabular}

die Frakturheilung nach 4-6 Monaten noch nicht abgeschlossen ist. Eine Pseudarthrose kann sich äußern in Form von Schmerzen im Bereich des betroffenen Kieferabschnitts, Rötung, Schwellung sowie eingeschränkter Funktion und Belastbarkeit. Außerdem kann eine abnorme Beweglichkeit des betroffenen Kieferabschnitts beobachtet werden. Unterschieden werden hier sog. schlaffe Pseudarthrosen mit deutlicher Beweglichkeit und straffe Pseudarthrosen, bei denen nur eine minimale Beweglichkeit vorliegt (vgl. $>$ Tab. 2).

\section{Grundsätze der Therapie}

Bei Pseudarthrosen mit erschöpfter osteogenetischer Potenz ist eine knöcherne Überbrückung nur dann zu erwarten, wenn die Voraussetzung zur Osteogenese durch chirurgische Maßnahmen geschaffen werden [7]. $\mathrm{Zu}$ den chirurgischen Maßnahmen zählen hier in der MKG-Chirurgie neben der Anfrischung der Stümpfe und der Überbrückung mit ortsständigen gestielten Knochentransplantaten [7] die Defektrekonstruktion mit freiem Spongiosa- oder Kortikospongiosatransfer bis hin zu ggf. mikrovaskulär anastomosierten Knochen- bzw. Knochenund Weichteiltransplantaten bei ausgedehnteren Defektsituationen (vgl. - Abb. 2).

Autologe Knochentransplantate stellen dabei aufgrund der guten osteoinduktiven Potenz den Goldstandard in der Pseudarthrosentherapie dar, sind aber mit einer entsprechenden Entnahmemorbidität verbunden. Das Grundprinzip der Pseudarthrosenresektion besteht in einer Exzision des Narbengewebes zwischen den Stümpfen, Deperiostierung und Resektion der kortikalen Deckschicht im Pseudarthrosenbereich und Osteoplastik [4]. Dies erfolgt i.d.R. mittels Auffüllung des Raumes zwischen den Stumpfenden mit Spongiosablöckchen (bei Spalten bis etwa $3 \mathrm{~mm}$ ) [7] bzw. mit Kortikospongiosablöcken in Verbindung mit einer stabilen Osteosynthese mit primär lasttragender Funktion. Liegen ausgedehnte Defektsituationen vor, ist im Narbengewebe bzw. in Arealen mit kompromittierter Gefäßsituation (sog. ersatzschwaches Lager [8]) mit Resorptionen des Transplantats zu rechnen, was den Einsatz z.B. homoioplastischer Transplantate bereits in der Vergangenheit einschränkte [7]. Im ersatzschwachen Lager wird bei ausgedehnten Defektsituationen bzw. Knochen-Weichteil-Defekten da- her heute mikrovaskulär anastomosierten Transplantaten der Vorzug gegeben [9].

Merke

Der klinische Erfolg bei der Therapie der Pseudarthrosen hängt dabei entscheidend von der Vaskularisierung und osteogenetischen Potenz von Lagergewebe und Transplantat sowie von der adäquaten Stabilität der Osteosynthese während der Einheilphase ab.

\section{Komplikationen in der Traumatologie}

\section{Epidemiologie zu Komplikationen}

Während die Pseudarthrose in der Ära der primär konservativen Kieferbruchbehandlung nach den Nervenverletzungen (1,4\%) mit 0,8\% die zweithäufigste unmittelbar frakturassoziierte Spätfolge noch vor Infektionen (0,5\%) und in Fehlstellung konsolidierten Frakturen $(0,4 \%)$ darstellte [10], hat sich das Komplikationsspektrum in der Traumatologie heute wesentlich verändert. Pseudarthrosen werden heute nur noch selten beobachtet. Im Bereich der Gelenkfortsatzfrakturen haben Pseudarthrosen sowohl nach konservativer als auch nach operativer Therapie (hier vor allem bei Versagen der Osteosynthese) allerdings weiterhin Bedeutung in der Traumatologie. Die Inzidenz für Komplikationen insgesamt nach Osteosynthese im kraniofazialen Bereich liegt heute bei 7,928,3\% [11-17]. Mandibulafrakturen stellen mit die häufigsten Brüche des Gesichtsschädels dar [16], somit ist die Studienlage über Komplikationen und mögliche Risikofaktoren gut. Die Komplikationsrate nach Mandibulafrakturen wird mit 7,9-21,2\% angegeben [11-13,15, $16,18]$, bei polytraumatisierten Patienten auf Intensivstation zwischen 11 und 28,3\% [14,17]. Die häufigsten Formen der Komplikationen sind posttraumatische bzw. postoperative Okklusionsstörungen $[13,15]$, Infektionen [13-15,18], extraorale Fistelbildung [13], freiliegendes Osteosynthesematerial $[13,18]$, Wunddehiszenzen $[15$, 18] sowie heute seltener Pseudarthrosen $[15,18]$ und Schädigung anatomischer Strukturen [15]. Diese Komplikationen, die ihrerseits wiederum das Risiko für das Auftreten von Pseudarthrosen erhöhen können, führen oftmals zu aufwendigen und mehrfach notwendigen resektiven und rekonstruktiven Eingriffen, welche die Rehabilitation der Patienten verlängern und erschweren. 

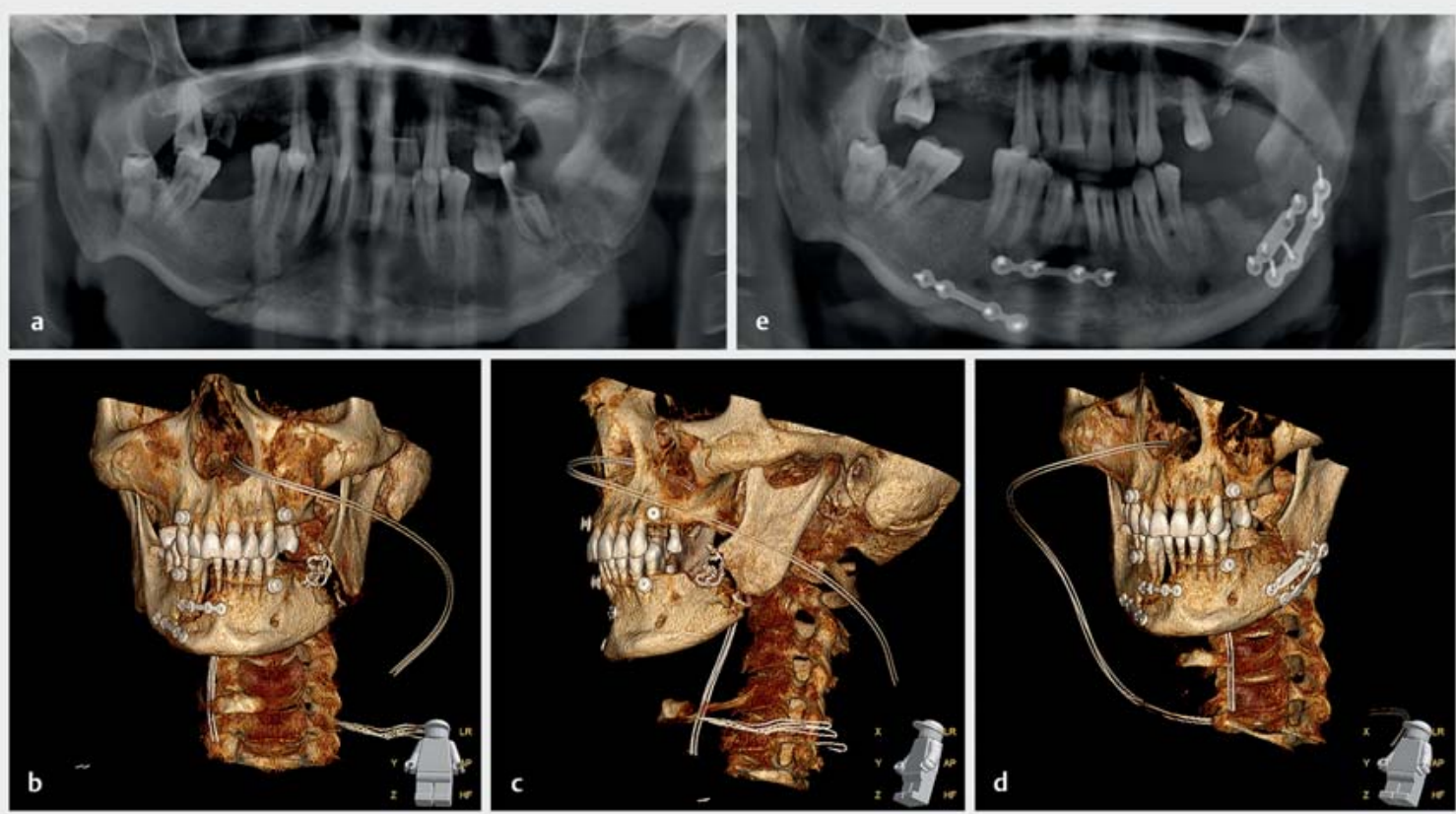

Abb. 3 a Orthopantomogramm: Patient mit veralteter doppelter Unterkieferfraktur; infizierte Pseudarthrose linker Kieferwinkel, Vorstellung wegen Abszessbildung perimandibulär links. b 3-D-CT: postoperative Kontrolle nach Osteosynthese der Paramedianfraktur rechts (2,0-mm-System), Streifeneinlage im Bereich der Pseudarthrose linker Kieferwinkel. c 3-D-CT: dito. Beachte die ausgedehnte knöcherne Defektsituation im linken Kieferwinkelbereich. d 3-D-CT: postoperative Kontrolle nach zweizeitiger Osteosynthese jetzt im linken Kieferwinkelbereich mit 2 verstärkten Locking-Platten des Systems $2,0 \mathrm{~mm}$ (grenzwertig stabile Versorgung). e Orthopantomogramm: postoperative Kontrolle 6 Wochen nach Osteosynthese, bis dato ungestörte Wundheilung und zunehmende Durchbauung der Frakturareale.

\section{Allgemeine Risikofaktoren für Komplikationen}

Das Auftreten von Komplikationen ist vielseitig und hängt - wie auch die Behandlung - von der Art der Komplikation und dem Bestehen möglicher Risikofaktoren ab. Alter, Hypertension, Dialyse und ASA-Klasse gelten als Risikofaktoren für allgemeine Komplikationen bei Osteosynthese $[16,17,19]$, zudem die Schwere der Fraktur [12], Zeit zwischen Verletzung und operativer Behandlung der Fraktur [18] und Anzahl der Operationen [17]. Polytraumapatienten auf der Intensivstation weisen bei mehr als einer Operation eine Erhöhung des Komplikationsrisikos um den Faktor 1,8 auf. Zudem steigt das Komplikationsrisiko um 4,5\% pro Lebensjahr [17]. Zu prüfen bleibt weiterhin, ob Faktoren wie Rauchen und Alkohol einen signifikanten Einfluss auf die Komplikationsrate darstellen [16]. Speziell für Infektionen nach Osteosynthese werden unabhängige Risikofaktoren wie junges Alter, penetrierende Verletzungen, Fraktur in mehreren Gesichtsdritteln, Gefäßverletzungen und operatives Management genannt [14]. Mit Ausnahme evtl. der Kieferwinkelfrakturen [11] besteht allerdings kein Zusammenhang zwischen der Komplikationsrate und der anatomischen Lokalisation des Bruches [16]. Im Gegensatz dazu wirkt sich die Zeit zwischen Frakturereignis und operativer Erstbehandlung mittels Osteosynthese signifikant auf das Auftreten von Komplikationen aus ( $\bullet$ Abb. 3). Bei Patienten ohne Komplikation erfolgte die operative Erstbehandlung im
Schnitt nach 4, bei Patienten mit Komplikation erst nach 6 Tagen [18].

\section{Spezielle Aspekte in der Traumatologie}

Speziell in der Traumatologie ist zu unterscheiden, ob eine - dann meist verzögerte - Bruchheilung durch Infektion (Bruchspaltostitis) bzw. diese fördernde bzw. unterhaltende Faktoren wie Sequesterbildung, Zahn- bzw. Wurzelreste im Bruchspaltbereich [3], endo- bzw. endoparodontale Läsionen bei dem Bruchspalt benachbarten Zähnen usw. unterhalten wird oder aber ggf. Pseudarthrosen auf große Knochendefekte, vorbestehende pathologische Knochenprozesse (z. B. Patienten nach Radiatio, unter Bisphosphonat- oder Antikörpertherapie, Zysten usw.) oder aber auf die Instabilität der gewählten Versorgung zurückzuführen sind. Während relativ frische Pseudarthrosen (3-6 Monate nach Trauma) bis in die 1980erJahre meist durch eine mehrmonatige (8-12 Wochen) straffe Immobilisation über dental oder mukosal abgestützte Schienungen therapiert wurden, konnte damit zwar in diesen frühen Pseudarthrosefällen noch eine gute Ausheilung erzielt werden $[3,4]$. Andererseits waren diese prolongierten straffen Immobilisationen unter parodontalhygienischen (Gingivitis, Parodontitis, Karies mit der Folge von Zahnverlust) sowie funktionellen Aspekten (Immobilisationsschäden am Kiefergelenk) hinsichtlich Spät- und Folgeschäden als problematisch zu bewerten 

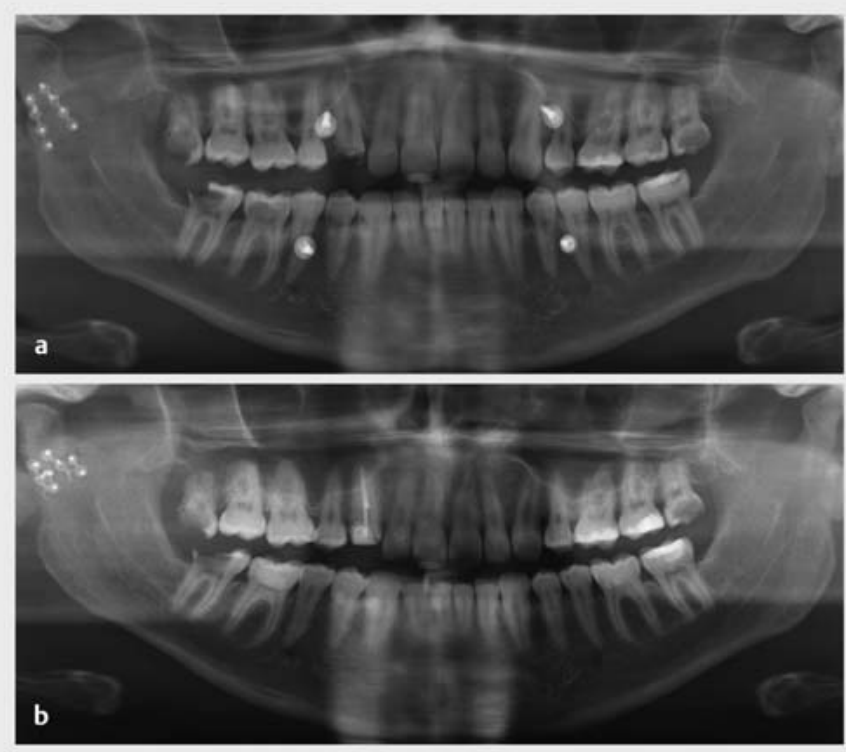

- Abb. 4 a Orthopantomogramm: Gelenkfortsatzbasisfraktur [20] rechts mit 2 miniaturisierten Platten (1,5-mm-System) bez. Stabilität suboptimal versorgt (Standard wären 2 2,0-mm-Platten oder 2,0-mm3-D-Platten). Beachte außerdem die Stufenbildung, die am Dorsalrand des Gelenkfortsatzes erkennbar ist, die Platten sind bei ungenauer Reposition somit mutmaßlich primär lasttragend. b Orthopantomogramm: Verlaufskontrolle 4 Monate nach Versorgung. Es zeigen sich Plattenbrüche und eine Sinterung des Gelenkfortsatzes, allerdings keine Pseudarthrosenbildung.

[11]. Die modernen operativen Verfahren mit Pseudarthroseresektion, Anfrischung, Knochentransfer und stabiler (Re-)Osteosynthese erfordern im Gegensatz dazu aus Gründen der Übersicht und zur Infektionsprophylaxe häufig invasive extraorale Zugänge. Dies geht einher mit den typischen zugangsbedingten Risiken für Narbenbildung und Nervenverletzungen und der Entnahmemorbidität für den Spongiosa- oder Kortikospongiosatransfer [20].

\section{Merke}

Insofern kommt der adäquaten und effizienten Primärbehandlung (stabile Osteosynthese und adäquates Weichgewebsmanagement) im Sinne einer Prävention der Pseudarthrosen hohe Bedeutung zu $[4,20]$.

\section{Wundinfektion und biomechanische Aspekte}

Eine der häufigsten verfahrensassoziierten Komplikation nach Plattenosteosynthese stellen Dehiszenzen mit Freiliegen der Osteosyntheseplatten dar mit Raten bis zu $19 \%$ [14], wobei hier sicherlich das Ausmaß der traumabedingten Schleimhautschädigung im individuellen Fall eine Rolle spielt [18]. Allerdings führt dies allein i.d.R. nicht zu Pseudarthrosen. Hierzu müssen weitere Faktoren wie Okklusionsstörungen bzw. eine instabile Okklusion und muskuläre Faktoren (z. B. Bruxismus) neben den wesentlichen biomechanischen Faktoren wie kompromittiertes Knochenangebot und/oder reduzierte interfrag- mentäre Abstützung im Frakturbereich zum Tragen kommen. Kommt es hierdurch zu einem Versagen der Osteosynthese mit der Folge anhaltender Hypermobilität im Frakturbereich, resultiert daraus in Arealen mit Kontakt zur Mundhöhle eine deutlich erhöhte Infektionsrate [11] mit der Folge einer Kontamination durch intraorale Keime. Dies betrifft den Frakturspalt, aber auch die eingebrachten Osteosynthesematerialien. Außerdem besteht eine hohe Wahrscheinlichkeit für das Auftreten einer Pseudarthrose infolge der kontinuierlichen Hypermobilität im Bruchspaltbereich. Die hohen funktionellen Kräfte, die durch die Kaumuskulatur, insbesondere an der Mandibula, zum Tragen kommen, sind geeignet, bei fehlender okklusaler Abstützung bzw. Okklusionsstörung osteosynthetische Versorgungen zum Versagen zu bringen, die unter normalen Verhältnissen eine ungestörte Knochenbruchheilung erlauben würden [20]. Klinische Hinweise für eine biomechanisch nicht ausreichend stabile Versorgung - sei es nun primär oder sekundär infolge Wundinfektion - sind gelockerte Schrauben [21] und/oder Plattenbrüche. Letztere können im weiteren Verlauf allerdings z.B. im Gelenkfortsatzbereich häufig problemlos ausheilen ( $\triangleright$ Abb.4). Es können aber auch sekundäre Dislokationen mit der Folge von postoperativen Fehlstellungen bis hin zum Auftreten einer Pseudarthrose auftreten (vgl. > Abb. 1).

Cave

Insbesondere bei verzögerter Versorgung oder Osteosyntheseversagen begünstigt die Kombination einer Infektion aufgrund einer intraoralen Dehiszenz in Kombination mit einer Hypermobilität der Fragmente das Auftreten einer Pseudarthrose nach Trauma.

Frakturen des atrophen Unterkiefers [22], Begleitfrakturen der Mandibula [23], speziell mehrfach fragmentierte Frakturen mit Beteiligung des Alveolarfortsatzes (Verlust der interdentalen Abstützung) sowie Kieferwinkelfrakturen mit nicht erhaltungsfähigem Zahn im Bruchspalt [11] stellen daher Prädilektionsstellen für eine Pseudarthrosenbildung dar ( $\triangleright$ Abb. 5). Die früher verwendeten Kompressionsplatten [11] sind heute zugunsten von 3-D-Plattensystemen weitgehend verlassen worden, die bei geringerer Dimension eine verbesserte biomechanische Stabilität liefern [24]. Allerdings sind neben Einflussfaktoren aufseiten der Behandler (Technikfehler bei der Schraubensetzung, Auswahl weniger geeigneter Osteosynthesesysteme etc.) [23, 25] auch Faktoren seitens der Patienten bei der Komplikationsanalyse nicht zu vernachlässigen, so z.B. eine kompromittierte Knochenqualität (vgl. - Abb. 1) oder mangelnde Compliance hinsichtlich Ernährungsform, Mundhygiene, Rauchen etc.

\section{Pseudarthrosen bei Gelenkfortsatzfrakturen}

Eine Sonderrolle hinsichtlich Pseudarthrosen besitzen die Gelenkfortsatzfrakturen, die mit etwa 25-30\% aller Mandibulafrakturen relativ häufig sind [21]. Da es sich i.d. R. 
um geschlossene Frakturen handelt, sind Bruchspaltinfektionen oder Fistelbildungen nach intra- oder extraoral im Regelfall nicht zu erwarten. Während bei konservativer Behandlung von Gelenkfortsatzfrakturen eine Heilung in Fehlstellung unter möglichst guter Wiederherstellung der Okklusion und Funktion angestrebt wird, zielt die operative Versorgung der Gelenkfortsatzfrakturen auf eine möglichst physiologische Wiederherstellung der anatomischen Strukturen hin [20]. Gerade nach konservativer Therapie treten häufiger Spätschäden in Form von Seitabweichungen bei den Kieferbewegungen aufgrund der in Fehlstellung verheilten Gelenkfortsatzfrakturen auf [26]. Die schwerwiegendste Komplikation ist die Ankylose, die meist nach Frakturen im Kindes- und Jugendalter auftritt, selten dagegen im Erwachsenenalter [10]. Hier kommt es im Gegensatz dazu bei unzureichender Kallusbildung oder Weichgewebsinterposition ggf. zu einer Pseudarthrosenbildung im Bereich des Gelenkfortsatzes. Dies erlaubt dann über die Rotation in der Pseudarthrose zwar eine meist zufriedenstellende Mundöffnung [26], ist jedoch meist mit einem zumindest partiellen Verlust der physiologischen Gelenkfunktion hinsichtlich Protrusion und Translation assoziiert. Insofern ist eine Pseudarthrosenbildung nach konservativer Versorgung somit funktionell eher als ein sogar erwünschter Kompensationsmechanismus zu verstehen. Im Gegensatz dazu wirkt sich ein - in dieser biomechanisch anspruchsvollen Frakturregion [6, 20, 21] nicht seltenes - Osteosyntheseversagen, z. B. bei Einsatz von einzelnen oder zu gering dimensionierten Miniplatten (vgl. > Abb. 1) mit anschließender Pseudarthrosenbildung nach operativer Frakturversorgung deutlich ungünstiger aus. Für Materiallockerungen besonders anfällig sind Gelenkhals- oder bilaterale Frakturen, z. B. bei fehlender okklusaler Abstützung [21]. Durch gelockertes Osteosynthesematerial kann es zu ausgeprägten Osteolysen mit Destruktion des Unterkieferastes bzw. Gelenkfortsatzes unter Ausbildung einer ausgedehnten Pseudarthrose kommen, die dann umfangreiche Rekonstruktionen bis hin zum totalen Kiefergelenkersatz erfordern [20].

Cave

Bei der operativen Frakturversorgung von Gelenkfortsatzfrakturen sind Pseudarthrosen im Gegensatz zur konservativen Therapie daher unbedingt zu vermeiden, da durch gelockerte Osteosynthesematerialien ausgedehnte Osteolysen induziert werden [20].

\section{Pseudarthrosen in der orthognathen Chirurgie}

Ramus- und LeFort-I-Osteotomien zur Korrektur von Dysgnathien zählen zu den am häufigsten durchgeführten elektiven Operationen im Bereich der MKG-Chirurgie. Die orthognathe Chirurgie mit ihren standardisiert gesetzten, regelhaft breiten Osteotomielinien unter Hei-
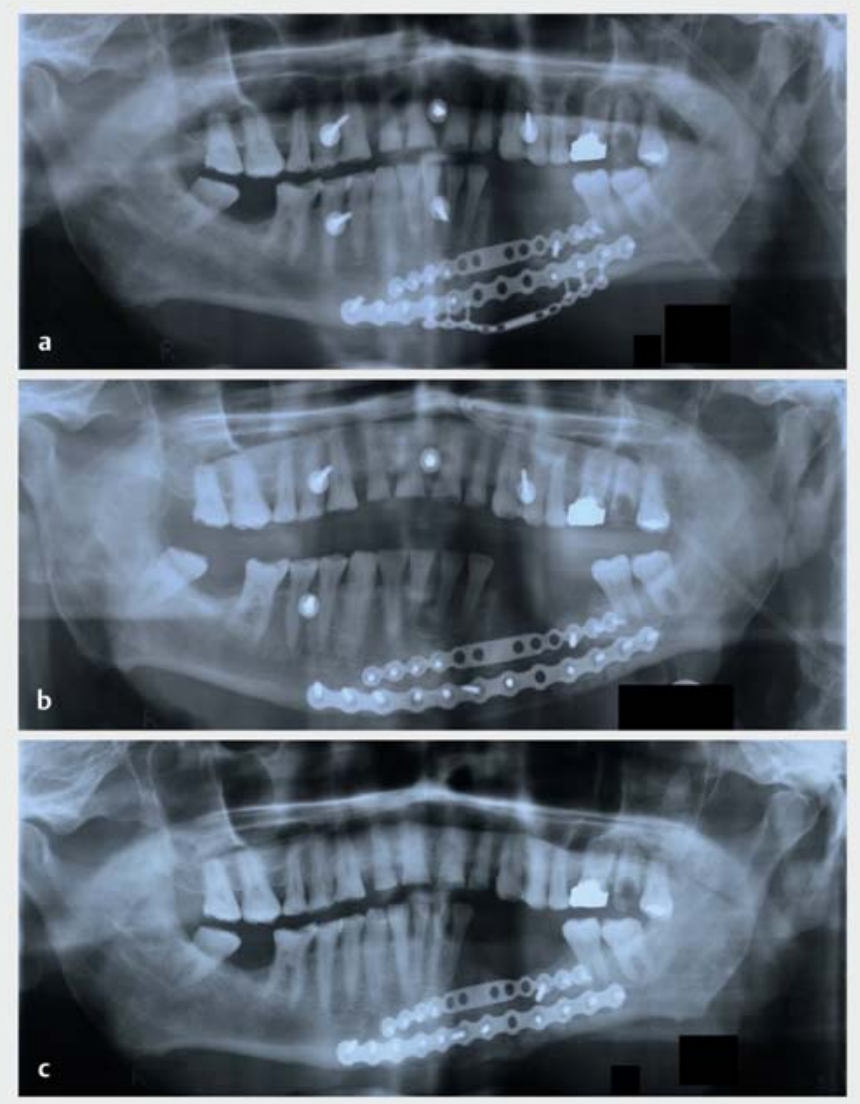

- Abb. 5 a Orthopantomogramm: Verlaufskontrolle nach Osteosynthese einer Unterkieferkorpusfraktur mit Majorfragmentation und Beteiligung der Transitionszone [5] links unter Verlust des Alveolarfortsatzes und der Zähne 33 bis 36. Nach Osteosynthese mit einer Traumaplatte (System 2,3 mm) und 2 Miniplatten (System 2,0 mm) sind Metallockerungen und Sequestierung erkennbar, klinisch besteht eine Hypermobilität im Sinne einer schlaffen Pseudarthrose. b Orthopantomogramm: Reosteosynthese mit Traumaplatte (System 2,3 mm) und Miniplatte (System 2,0 mm) nach Sequestrotomie, Auffüllung des Defektbereichs mit autologer Spongiosa unter plastischem Wundverschluss. Anschließend Ruhigstellung über intermaxilläre Fixationsschrauben und Elastics. c Orthopantomogramm: Verlaufskontrolle, die ehemalige Pseudarthrose zeigt bei diesmal infektionsfreiem Verlauf eine zunehmende knöcherne Konsolidierung.

lung i. d. R. ohne breite Knochenkontaktflächen ist ein exzellentes Beispiel dafür, dass der Stabilität der Osteosynthese eine wesentliche Bedeutung für die Vermeidung von Pseudarthrosen zukommt. Intraoperative Komplikationen bei der orthognathen Chirurgie betreffen heute in erster Linie Störungen der Sensibilität des N. alveolaris inferior, Blutverlust und sog. „Bad Splits“ [27]. Infektionen und Pseudarthrosen sind seit Einsatz moderner Osteosynthesesysteme dagegen selten. Selbst in 2 großen Serien von insgesamt knapp 4000 orthognathchirurgischen Eingriffen $[28,29]$ wird nicht über Pseudarthrosen berichtet, die Rate an freiliegendem Osteosynthesematerial ist mit 0,2\% sehr niedrig [29]. Wesentliche Unterschiede zum Traumakollektiv sind demnach die Traumaintensität, hier mit geringer Affektion des Weichteil- 

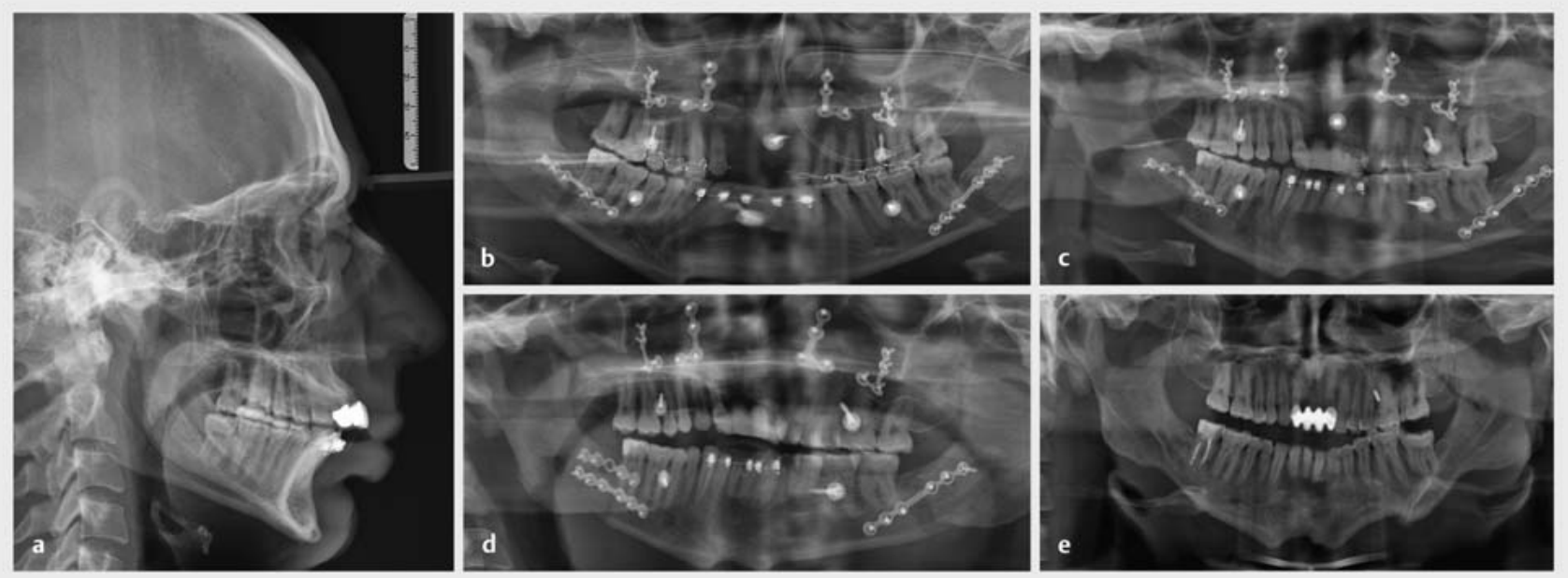

- Abb. 6 a Fernröntgenseitenbild: Ausgangsbefund bei Dysgnathie der Klasse Angle II (Distalbisslage) vor orthognather Umstellungsoperation. b Orthopantomogramm: postoperative Kontrolle mit korrekter skeletaler Fragmentstellung und Plattenposition. Beachte den nur punktuellen Kontakt des rechten Seitenzahnbereichs in mutmaßlich suboptimaler, instabiler Verzahnung. c Orthopantomogramm: Fraktur der Platte im rechten Unterkieferwinkel, das proximale Fragment (Unterkieferast) steht rotiert. Beachte den jetzt infolge Fraktur der Platte möglichen stabileren Zahnkontakt (instabile Okklusion damit verbessert). d Orthopantomogramm: Nach Reosteosynthese steht das kleine Fragment rechts wieder korrekt. Beachte die Disklusion mit jetzt wieder gemäß präoperativer Planung reduziertem Zahnkontakt rechts, der kieferorthopädisch eingestellt werden muss. e Orthopantomogramm: Nach Metallentfernung 6 Monate post operationem ist der rechte Kieferwinkel gut durchbaut (Nebenbefund: frakturierter Schraubenrest im Oberkiefer links).

mantels. Außerdem wird im Rahmen der orthognathen Chirurgie i.d.R. die Muskulatur umfangreich abgelöst. Konsekutiv liegt also in der orthognathen Chirurgie eine deutlich reduzierte Kaukraft während der Heilungsphase vor. Im Zeitalter der Drahtnahtosteosynthese lag die Rate an echten Pseudarthrosen dagegen noch bei etwa 1:100 bis $1: 300$ [2]. Infektionen aufgrund instabiler Fragmentstellung führten damals unweigerlich zum Misserfolg bzw. Rezidiv, selbst eine Heilung in Fehlstellung konnte nicht erwartet werden [2]. In schwierigen Fällen, wie z. B. sehr weiten Verlagerungsstrecken oder bei Narbenbildungen im Rahmen von Lippen-Kiefer-Gaumen-Spalten, die früher mit einer hohen Rezidivgefahr verbunden waren [30], kann heute eine Distraktionsosteogenese diese Komplikation vermeiden. Trotzdem sei an die unter dem Abschnitt Traumatologie diskutierten Grenzen der Osteosynthese erinnert, die auch nach orthognather Chirurgie Geltung besitzen. So können z. B. bei einer instabilen Okklusion in Kombination mit Bruxismus und/oder Habits die sonst zuverlässigen Osteosynthesen an ihre Grenzen gelangen und somit einer Pseudarthrose Vorschub leisten ( $\triangleright$ Abb. 6).

\section{Merke}

Bei geringer Knochenanlagerungsfläche oder Osteosynthesen auf Distanz (counter-clockwise-Rotation) ohne simultane Knochenaugmentation muss in der orthognathen Chirurgie daher auch heute noch mit der Möglichkeit von Pseudarthrosen gerechnet werden.

\section{Pseudarthrosen in der rekonstruktiven Chirurgie}

\section{Autologe freie Knochentransplantate}

Während in der Traumatologie (verbesserte Osteosynthesesysteme) und orthognathen Chirurgie (verbesserte Osteosynthesesysteme und gutes Weichteillager) die Bedeutung der Pseudarthrosen deutlich abgenommen hat, sind Pseudarthrosen in der rekonstruktiven Chirurgie trotz des verbreiteten Einsatzes mikrovaskulärer Transplantate ein hoch relevantes Problem. Die früher verwendeten freien Knochentransplantate stoßen bei langstreckigen Defekten an ihre Grenzen, da sie keinen simultanen Weichgewebstransfer erlauben und im Falle einer postoperativen Radiatio häufig verloren gehen [12]. Generell gilt, dass für die Komplikationsrate bei freien Transplantaten (Beckenkamm, Rippe, sternoklavikulares Transplantat usw.) zur Sekundärrekonstruktion Faktoren wie Radiatio, Nikotin- und Alkoholabusus und ein ersatzschwaches Weichteil- und Knochenlager relevant sind [31]. Im ersatzschwachen Lager ist das Endstromgebiet so stark kompromittiert, dass das Gewebe meist nicht in der Lage ist, das Transplantat adäquat zu ernähren und drohende Infektionen abzuwehren. Die Verlustrate ist bei jüngeren Patienten und Indikation für benigne Tumoren (d. h. keine Radiatio erfolgt) daher deutlich günstiger [31]. Seit Etablierung der mikrovaskulären Transplantate werden dagegen freie Knochentransplantate in der Tumorchirurgie nur noch sehr zurückhaltend eingesetzt und 
i.d.R. nur bei kleinstreckigen Defektrekonstruktionen, benignen bzw. nicht bestrahlten Tumoren verwendet. Die Erfolgschance für freie Transplantate kann gesteigert werden, wenn die Rekonstruktionen ggf. in multiplen Einzelschritten erfolgen [9, 32]. Eine Option zur Rekonstruktion ausgedehnter Defekte bietet in diesem Kontext bei ersatzstarkem Lager auch die Distraktionsosteogenese bzw. Transportdistraktionsosteogenese. Der Vorteil der Transportdistraktionsosteogenese liegt in der simultanen Augmentation der Weichgewebe. Die Rate an Pseudarthrosen bzw. Nonunion-Komplikationen wird in einer Fallserie mit 2/14 (14\%) angegeben, stellt somit auch für die Distraktionsosteogenese ein potenzielles Problem dar [32]. Die Therapie ist in diesem Fall - wie in der Traumatologie - die Anfrischung mit Spongiosatransfer und funktionsstabiler Osteosynthese.

\section{Mikrovaskuläre Rekonstruktionen}

\section{Epidemiologie zu Pseudarthrosen}

Für mikrovaskulär anastomosierte Rekonstruktionen, für die die Problematik des ersatzschwachen Lagers nur in eingeschränkter Weise gilt [31], wird die Rate an Pseudarthrosen in der älteren Literatur noch mit etwa 7-9\% angegeben $[12,17]$. Gemäß der aktuellen Review-Literatur liegt die Rate bei etwa 5\%, wobei hier laut Autoren vermutlich ein „überraschend hoher Reporting Bias vorliegt, zumal die damit verbundene Morbidität relevant ist“ [12]. Unabhängig von der verwendeten Osteosynthese (Miniplatten vs. Rekonstruktionsplatten) wurde für segmentale Defekte bei Fibulatransplantaten über eine Rate an Pseudarthrosen (Mal-/Nonunion) von sogar bis zu 14 bzw. 13\% berichtet. Plattenbrüche traten dabei zwar bei Miniplattenosteosynthesen deutlich häufiger auf (10 vs. $0 \%)[17,19]$, die Problematik liegt demnach aber vorwiegend im ersatzschwachen Lager und in adjuvanten Therapien (Radiatio, Radiochemotherapie) begründet. Auch hängt die Rate an Pseudarthrosen signifikant vom verwendeten Transplantattyp ab. Das Fibulatransplantat ist das derzeit am meisten verwendete Transplantat für die Mandibularekonstruktion und erlaubt eine mehrfache Segmentierung, also multiple Osteotomien zur optimalen Formanpassung [17, 33, 34]. Die Rate an Transplantatversagen insgesamt liegt gemäß systematischem Review (9499 Defekte) für die Fibula bei 4,1\% und ist für den Beckenkamm mit 6,2\% am höchsten [12], wenngleich eine weitere Metaanalyse im Widerspruch dazu keine relevanten Unterschiede zwischen Beckenkamm und Fibula sieht [35]. Weitere mögliche Komplikationen sind Plattenbruch, Fistelbildung, Knochennekrose und Pseudarthrosen $[27,36]$. Die Rate an Pseudarthrosen hängt hier statistisch signifikant vom verwendeten Transplantat ab. Osteokutane Radialislappen und Skapulalappen weisen gemäß Review mit 9,1\% (78/866) bzw. 13,1\% (49/375) deutlich höhere Pseudarthroseraten auf als die Fibulatransplantate mit 3,9\% (103/2632). Straffe Pseudarthrosen ohne klinischen Interventionsbedarf werden für Skapulatransplantate sogar in bis zu 39\% der Rekonstruktio- nen berichtet. Die Beckenkammtransplantate schneiden hier mit 2,6\% (17/646) am besten ab [12]. Bezüglich der in der Literatur berichteten Raten ist allerdings zu bedenken, dass nur eine 3-dimensionale Bildgebung (CT, DVT [DVT: digitales Volumentomogramm] im Gegensatz zum Orthopantomogramm) eine zuverlässige Aussage über das Ausmaß einer Knochenheilungsstörung geben kann [33]. Orthopantomogrammbefunde unterschätzen demnach die Inzidenz von Pseudarthrosen.

\section{Risikofaktoren für Pseudarthrosen in der rekonstruktiven Chirurgie}

Als Risikofaktoren wurden Alter, Defektgröße, Radiotherapie und Rauchen einbezogen, wobei sich nur die Radiotherapie und das Rauchen als signifikante Risikofaktoren herausstellten [27,37].

\section{Merke \\ Die Komplikationsrate für Infektionen, freiliegende Platten und Pseudarthrosen ( $\triangleright$ Abb. 7) steigt bei be- strahlten Patienten signifikant an [19]. Andererseits ist eine ungestörte Knochenheilung Voraussetzung für den Start einer Strahlentherapie [33].}

Aus diesem Grund wird nach Rekonstruktion eine engmaschige Kontrolle nach Radiatio über einen Zeitraum von mindestens 6-16 Monaten vor etwaigen weiteren Maßnahmen empfohlen [19]. Die Komplikationsraten insgesamt werden mit $28 \%$ angegeben, es finden sich aber Arbeiten, die - abhängig von der Definition - über gewichtete Komplikationsraten bis zu $48 \%$ berichten, wobei die Rate an Knochennekrosen bei Verwendung von Rekonstruktionsplatten im Gegensatz zu Miniplatten möglicherweise höher zu sein scheint. Hierfür kommen möglicherweise Stress-Shielding-Effekte bei rigiden Rekonstruktionsplatten als Ursache infrage, was auch Relevanz für die Ausbildung von Osteoradionekrosen haben könnte [17]. Auch die Möglichkeiten der Konfiguration von Fibulatransplantaten zur Defektrekonstruktion hängt neben der Defektgröße wiederum entscheidend von einer Strahlentherapie ab. So wird aufgrund hoher Komplikationsraten bei der Versorgung von Typ-H-Defekten (vgl. - Tab. 3) bei bestrahlten Patienten explizit davon abgeraten, eine Rekonstruktion des Kondylus mit einer Gelenkprothese durchzuführen. Chirurgische Optionen wie z.B. Double-Barrel-Fibularekonstruktionen, die die Stabilität erhöhen könnten [38] und eine dentale implantatgetragene Rehabilitation der Patienten erleichtern würden, sind bei langstreckigen H-Defekten somit i.d. R. bei bestrahlten Patienten a priori nicht möglich [39]. Außerdem führt dies wiederum dazu, dass die Kieferwinkelregion mit Gelenkfortsatz autolog rekonstruiert werden muss. Die geringe Knochenanlagerungsfläche macht diese biomechanisch besonders sensible Region wiederum für Pseudarthrosen besonders anfällig ( $\mathbf{A} \mathbf{A b} \mathbf{b}$. 8). In einer radiologischen Follow-up-Studie konnte bei allen 5/56 Fibulae (9\%) mit Pseudarthrose diese jeweils im Kiefer- 

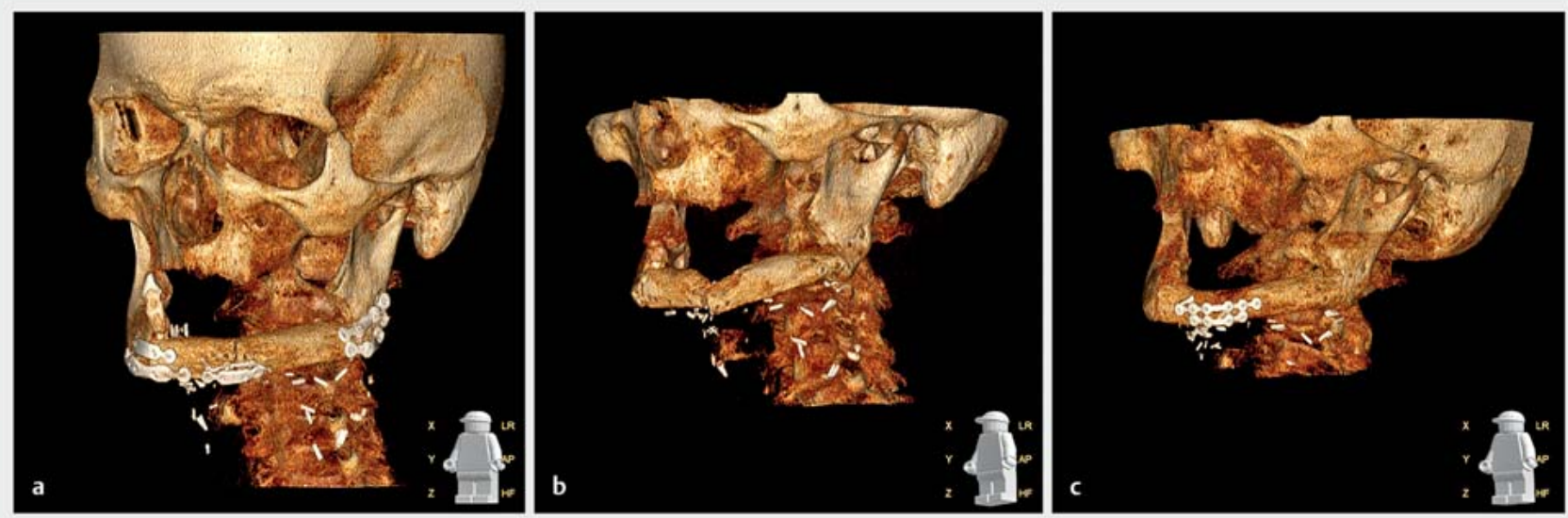

Abb. 7 a 3D-CT Serie: Bei Z.n. Fibularekonstruktion wurde eine nach Strahlentherapie krestal freiliegende Platte (linker Eckzahnbereich) vorzeitig entfernt, hier liegt eine Gentamycinkette auf. b 3-D-CT: sekundäre Fragmentdislokation aufgrund Pseudarthrose (die übrigen Osteotomiestellen sind nach erfolgter Metallentfernung gut verheilt). Beachte die Resorption im Bereich der ehemals freiliegenden Platte. c 3-D-CT: Kontrolle nach Anfrischung und Reosteosynthese.

- Tab. 3 Defekttypen der Mandibula nach dem HCL-System [41].

\begin{tabular}{|l|l|}
\hline $\begin{array}{l}\text { Defekttyp nach } \\
\text { Jewer et al. [41] }\end{array}$ & Beschreibung \\
\hline H-Defekt & $\begin{array}{l}\text { lateraler Defekt jeglicher Länge, inklusive Kondylus, } \\
\text { aber ohne relevante Mittellinienüberschreitung }\end{array}$ \\
\hline L-Defekt & $\begin{array}{l}\text { lateraler Defekt jeglicher Länge ohne relevante Mit- } \\
\text { tellinienüberschreitung, ohne Kondylusbeteiligung }\end{array}$ \\
\hline C-Defekt & $\begin{array}{l}\text { die Defekte betreffen das gesamte Kinnsegment } \\
\text { einschließlich aller Schneidezähne und Eckzähne }\end{array}$ \\
\hline
\end{tabular}

winkel festgestellt werden [33]. Gemäß FE-Analysen (FE: Finite Elemente) liegen die Van-Mises-Stresswerte im Kieferwinkelbereich von Fibularekonstruktionen 5-mal höher als im vergleichbaren normalen Knochen [33]. Dass hier nicht noch häufiger Pseudarthrosen auftreten, dürfte demnach auf die deutlich eingeschränkte muskuläre Funktion nach ablativer Tumorchirurgie und ggf. Radiatio zurückzuführen sein [40].

Biomechanische Aspekte

Speziell die Mandibula unterliegt hohen biomechanischen Belastungen aufgrund des komplexen Bewegungsmusters (Protrusion und Translation) beim Kauen, Sprechen und Schlucken [42]. Nach ablativer Chirurgie und Rekonstruktion besteht seitens der Patienten eine hohe Erwartungshaltung bez. dentaler Rehabilitation, die jedoch insbesondere nach Strahlentherapie zu Weichteilproblemen und somit Komplikationen führen kann. Eine strahleninduzierte Xerostomie muss hier als wichtiger Kofaktor gesehen werden [42].
Merke

Die Prävention einer Pseudarthrose durch stabile Osteosynthese und bestmögliche Rekonstruktion der Hart- und Weichgewebe muss somit bereits bei der Planung der Rekonstruktion im Vordergrund stehen.

Zu prüfen bleibt gemäß aktueller Literatur diesbezüglich weiterhin, ob die Art und Anzahl der verwendeten Osteosyntheseplatten einen signifikanten Einfluss auf die Komplikationsrate darstellen [27,37]. Eine rein auf Miniplatten basierte Osteosynthese ist dabei signifikant mit höheren Komplikationen, wie z. B. freiliegenden Platten, assoziiert. Diese Komplikation tritt gehäuft nach prä- und/ oder postoperativer Bestrahlung auf [19] (vgl. > Abb. 7). Außerdem könnten die zunehmend eingesetzten patientenspezifischen Implantate und der Einsatz von CAD-/ CAM-Techniken für Cutting Guides durch verbesserte Knochenadaptation die Komplikationsrate für Pseudarthrosen senken. Nachdem die Pseudarthrose in der rekonstruktiven Chirurgie aufgrund der damit verbundenen hohen Morbidität [12] ein klinisch relevantes Problem darstellt, stellt sich aus klinischer Sicht außerdem die Frage, inwieweit hier biomechanische Faktoren wie Defektgeometrie (vgl. - Tab.3) oder aber technische Einflussgrößen wie die Gestaltung des Übergangs zwischen den Knochenfragmenten (z. B. Schaffung von Anlageflächen durch Ostektomien) für die Entstehung bzw. Prävention von Pseudarthrosen eine Rolle spielen. Die Beurteilung bzw. Kontrolle einer erfolgreichen Knochenheilung sollte dabei bevorzugt mittels CT oder DVT erfolgen, die im Gegensatz zum 2-dimensionalen Orthopantomogramm (Schichtaufnahme) eine Beurteilung der Überbrückung der vestibulären und lingualen kortikalen Kontaktzonen erlaubt. Eine 3-D-Bildgebung ist daher insbesondere vor dentalen Rehabilitationen, z. B. mittels Implantaten, als 

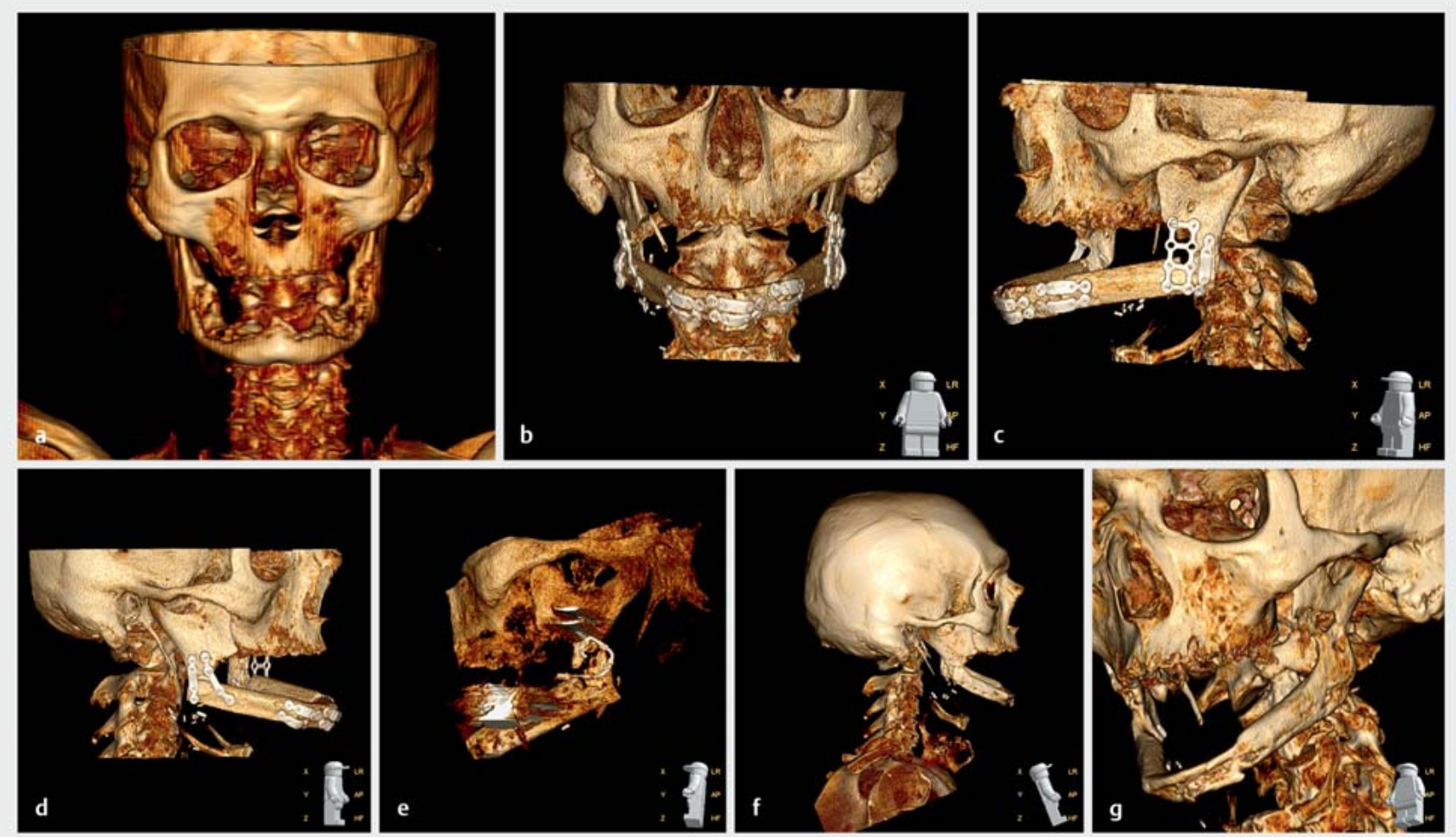

- Abb. 8 a 3-D-CT: Ausgangsbefund mit subtotaler Destruktion der Mandibula bei Osteoradionekrose. b 3-D-CT: Unterkieferrekonstruktion mit Fibula, Osteosynthese mit 2,0-mm-Locking-Platten im Fontbereich und 2,0-mm- bzw. 2,3-mm-Locking-Platten im Kieferwinkelbereich beidseits bei LCL-Defekt. c 3-D-CT: Versorgung des linken Kieferwinkels mit 3-D- und 2-Loch-Locking-Platten (System 2,0 mm). d 3-D-CT: Versorgung des rechten Kieferwinkels mit 2 Locking-Platten (Systeme 2,0 und 2,3 mm). e CT-Schichtaufnahme: Pseudarthrose im Bereich des linken Kieferwinkels, Nonunion mit straffer Pseudarthrose. f 3-D-CT: Kontrolle 3 Jahre nach Mandibularekonstruktion zeigt gute Ossifikation im Bereich des rechten Kieferwinkels. g 3-D-CT: dito, nach zwischenzeitlich erfolgter Reosteosynthese jetzt auch suffiziente Knochenbrücke im Bereich des linken Kieferwinkels.

obligat anzusehen [33]. Sowohl für Mandibularekonstruktionen $[43,44]$ als auch für segmentale Oberkieferdefekte [45] ist die Datenlage zu Implantatversorgungen derzeit allerdings noch relativ spärlich.

\section{Merke}

Vor dentaler Implantation wird die Entfernung des im Rahmen der Rekonstruktion eingebrachten Osteosynthesematerials empfohlen [33]. Eine evtl. vorliegende Pseudarthrose muss für die Festlegung des OPKonzepts vorab abgeklärt werden.

\section{Fazit}

Auch wenn die Ursachen für Pseudarthrosen multifaktoriell sind, bietet eine gute Weichteildeckung in Verbindung mit einer stabilen Osteosynthese die beste Erfolgschance für eine ungestörte Knochenheilung. Bei bestrahlten Patienten ist eine Pseudarthrose nach Rekonstruktion ein Zeichen entweder für eine schlechte Vaskularisierung des Knochens (ortsständig und/oder Transplantat) oder einen inadäquaten Knochenkontakt, weshalb dem Erhalt der Vaskularisierung des erhaltenen Mandibulaanteils und einer effizienten Osteosynthese speziell im Kieferwinkelbereich hohe Bedeutung zukommt [33]. Patientenspezifische Implantate und CAD-/CAM-Techniken erlauben eine Optimierung der Knochenanlagerungsflächen und lassen in Zukunft eine Reduzierung der Pseudarthroseraten erwarten.

\section{Interessenkonflikt}

Design Surgeon bei der Firma Medartis, Basel, Ch (Beratervertrag); Wissenschaftliche Vorträge für die Firma Karl Storz, Tuttlingen, D; Board Mitglied der IBRA, Basel, Ch, Head Educational Board mit wissenschaftlichen Vorträgen und Publikationen; Board Mitglied der SORG, Head TMJ Section mit wissenschaftlichen Vorträgen und Publikationen; Präsident der ESTMJS mit Industriesponsoring durch Medartis und KLS Martin, Storz, TMJ Concepts und Zimmer Biomet 2018 und 2018 als Kongresspräsident 


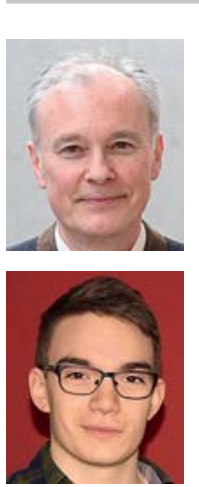

\section{Andreas Neff}

Univ.-Prof. Dr. Dr., Direktor der Klinik und Poliklinik für Mund-, Kiefer- und Gesichtschirurgie, Universitätsklinikum Marburg, UKGM GmbH

\section{Jonas Jung}

cand. med., Doktorand, Klinik und Poliklinik für Mund-, Kiefer- und Gesichtschirurgie, Universitätsklinikum Marburg, UKGM GmbH
Korrespondenzadresse

Univ.-Prof. Dr. Dr. med. Andreas Neff

Klinik und Poliklinik für Mund-, Kiefer- und Gesichtschirurgie Universitätsklinikum Marburg

Baldingerstraße

35043 Marburg

Tel.: $06421 / 5863237$

Fax: 06421/5863237

neffa@med.uni-marburg.de

\section{Literatur}

[1] Champy M, Loddé JP, Schmitt R et al. Mandibular osteosynthesis by miniature screwed plates via a buccal approach. J Maxillofac Surg 1978; 6: 14-21

[2] Steinhäuser EW. Infekt und Pseudarthrose als Komplikation nach selektiven Ramusosteotomien. In: Pfeifer G, Schwenzer N, Hrsg. Fortschritte der Kiefer- und Gesichts-Chirurgie: Band XXX: Aufklärung, Fehler und Gefahren in der Mund-Kiefer-Gesichts-Chirurgie, Erkennung und Behandlung. Stuttgart New York: Thieme; 1985: 88-91

[3] Francksen U. Zur konservativen Behandlung der UnterkieferPseudarthrosen. In: Schuchardt K, Wassmund M, Hrsg. Fortschritte der Kiefer- und Gesichts-Chirurgie: Ein Jahrbuch. Band II. Stuttgart: Thieme; 1956: 41-43

[4] Spiessl B, Günther H, Lentrodt J. Die Behandlung von Pseudarthrosen des Unterkiefers mit autogenem Rippenknochen. In: Schuchardt K, Hrsg. Fortschritte der Kiefer- und GesichtsChirurgie: Band XII: Die Therapie der Spätfolgen nach Traumen im Kiefer-Gesichts-Bereich. Stuttgart: Thieme; 1967: 215-219

[5] Cornelius C-P, Kunz C, Neff A et al. The Comprehensive AOCMF Classification System: fracture case collection, diagnostic imaging work up, AOCOIAC iconography and coding. Craniomaxillofac Trauma Reconstr 2014; 7 (Suppl. 1): S131135. doi: $10.1055 / \mathrm{s}-0034-1393722$

[6] Neff A, Cornelius C-P, Rasse M et al. The Comprehensive AOCMF Classification System: condylar process fractures - level 3 tutorial. Craniomaxillofac Trauma Reconstr 2014; 7 (Suppl. 1): S044-S058. doi:10.1055/s-0034-1389559

[7] Schüle $\mathrm{H}$. Experimentelle und klinische Untersuchungen zur Bewertung proteinarmer Knochenspäne für die Pseudarthrose-Behandlung. In: Pfeifer G, Schwenzer N, Hrsg. Fortschritte der Kiefer- und Gesichts-Chirurgie: Band XXX: Aufklärung, Fehler und Gefahren in der Mund-Kiefer-Gesichts-Chirurgie, Erkennung und Behandlung. Stuttgart, New York: Thieme; 1985: 205-214
[8] Lexer E. Die Verwendung der freien Knochenplastik. Verh Dtsch Ges Chir 1908; 2: 188-203

[9] Guerrier G, Alaqeeli A, Al Jawadi A et al. Reconstruction of residual mandibular defects by iliac crest bone graft in warwounded Iraqi civilians, 2006-2011. Br J Oral Maxillofac Surg 2015; 53: e27-e31. doi:10.1016/j.bjoms.2012.06.003

[10] Müller W. Häufigkeit und Art der Spätfolgen bei 2733 Frakturen des Gesichtsschädels. In: Schuchardt K, Hrsg. Fortschritte der Kiefer- und Gesichts-Chirurgie: Band XII: Die Therapie der Spätfolgen nach Traumen im Kiefer-Gesichts-Bereich. Stuttgart: Thieme; 1967: 220-224

[11] Ardary WC. Prospective clinical evaluation of the use of compression plates and screws in the management of mandible fractures. J Oral Maxillofac Surg 1989; 47: 1150-1153

[12] Brown JS, Lowe D, Kanatas A et al. Mandibular reconstruction with vascularised bone flaps: a systematic review over 25 years. Br J Oral Maxillofac Surg 2017; 55: 113-126. doi:10.1016/j.bjoms.2016.12.010

[13] Daar DA, Kantar RS, Cammarata MJ et al. Predictors of adverse outcomes in the management of mandibular fractures. J Craniofac Surg 2019; 30: 571-577. doi:10.1097| SCS.0000000000005195

[14] Daif ET. Correlation of plates' number with complications of osteosynthesis in mandibular fractures. J Craniofac Surg 2014; 25: e526-e529. doi:10.1097/SCS.0000000000001038

[15] Hsieh T-Y, Funamura JL, Dedhia R et al. Risk factors associated with complications after treatment of mandible fractures. JAMA Facial Plast Surg 2019. doi:10.1001/jamafacial.2018.1836

[16] Kantar RS, Cammarata M], Rifkin W] et al. Bimaxillary orthognathic surgery is associated with an increased risk of early complications. J Craniofac Surg 2019; 30: 352-357. doi:10.1097/SCS.0000000000005026

[17] Robey AB, Spann ML, McAuliff TM et al. Comparison of miniplates and reconstruction plates in fibular flap reconstruction of the mandible. Plast Reconstr Surg 2008; 122: 1733-1738. doi:10.1097/PRS.0b013e31818a9ac5

[18] Edwards T], David DJ, Simpson DA et al. The relationship between fracture severity and complication rate in miniplate osteosynthesis of mandibular fractures. Br J Plast Surg 1994; 47: 310-311

[19] Chen Y, Wu J, Gokavarapu S et al. Radiotherapy and smoking history are significant independent predictors for osteosynthesis-associated late complications in vascular free fibula reconstruction of mandible. J Craniofac Surg 2017; 28: 15081513. doi: $10.1097 /$ SCS. 0000000000003704

[20] Neff A. Open reduction and internal fixation in temporomandibular joint traumatology: current concepts and future perspectives. Stomatological Dis Sci 2019; 3: 2. doi:10.20517| 2573-0002.2018.27

[21] Sikora M, Sielski M, Stąpor A et al. Use of the Delta plate for surgical treatment of patients with condylar fractures. J Craniomaxillofac Surg 2016; 44: 770-774. doi:10.1016/j. jcms.2016.04.008

[22] Flores-Hidalgo A, Altay MA, Atencio IC et al. Management of fractures of the atrophic mandible: a case series. Oral Surg Oral Med Oral Pathol Oral Radiol 2015; 119: 616-627. doi:10.1016/j.oooo.2015.01.016

[23] Chrcanovic BR. Fixation of mandibular angle fractures: clinical studies. Oral Maxillofac Surg 2014; 18: 123-152. doi:10.1007/ s10006-012-0374-1

[24] Kanubaddy SR, Devireddy SK, Rayadurgam KK et al. Management of Mandibular Angle Fractures: Single Stainless Steel Linear Miniplate Versus Rectangular Grid Plate-A Prospective 
Randomised Study. J Maxillofac Oral Surg 2016; 15: 535-541. doi:10.1007/s12663-016-0892-8

[25] Khandeparker PVS, Dhupar V, Khandeparker RVS et al. Transbuccal versus transoral approach for management of mandibular angle fractures: a prospective, clinical and radiographic study. J Korean Assoc Oral Maxillofac Surg 2016; 42: 144150. doi:10.5125/jkaoms.2016.42.3.144

[26] Becker R. Die Abhängigkeit der Spätergebnisse von der Verletzungsart und der Behandlung, untersucht an 318 Fällen von Verletzungen des Gesichtsschädels. In: Schuchardt K, Hrsg. Fortschritte der Kiefer- und Gesichts-Chirurgie: Band XII: Die Therapie der Spätfolgen nach Traumen im Kiefer-Gesichts-Bereich. Stuttgart: Thieme; 1967: 225-231

[27] Olate S, Sigua E, Asprino L et al. Complications in orthognathic surgery. J Craniofac Surg 2018; 28: e158-e161. doi:10.1097| SCS.0000000000004238

[28] Chow LK, Singh B, Chiu WK et al. Prevalence of postoperative complications after orthognathic surgery: a 15-year review. J Oral Maxillofac Surg 2007; 65: 984-992. doi:10.1016/j. joms.2006.07.006

[29] Robl MT, Farrell BB, Tucker MR. Complications in orthognathic surgery: a report of 1,000 cases. Oral Maxillofac Surg Clin North Am 2014; 26: 599-609. doi:10.1016/j.coms.2014.08.008

[30] Hörster W, Beyer J-D. Vermeidung unerwünschter Ergebnisse bei Osteosynthese der Maxilla im Rahmen der kieferorthopädischen Chirurgie. In: Pfeifer G, Schwenzer N, Hrsg. Fortschritte der Kiefer- und Gesichts-Chirurgie: Band XXX: Aufklärung, Fehler und Gefahren in der Mund-Kiefer-GesichtsChirurgie, Erkennung und Behandlung. Stuttgart New York: Thieme; 1985: 100-102

[31] Riediger D. Probleme bei der Osteosynthese des frei und mikrochirurgisch transplantierten Knochens. In: Pfeifer G, Schwenzer N, Hrsg. Fortschritte der Kiefer- und Gesichts-Chirurgie: Band XXX: Aufklärung, Fehler und Gefahren in der Mund-Kiefer-Gesichts-Chirurgie, Erkennung und Behandlung. Stuttgart New York: Georg Thieme; 1985: 172-175

[32] Zwetyenga N, Siberchicot F, Emparanza A. Reconstruction of large mandibular and surrounding soft-tissue defects using distraction with bone transport. Int J Oral Maxillofac Surg 2012; 41: 1215-1222. doi:10.1016/j.jom.2012.03.020

[33] Akashi M, Hashikawa K, Kakei Y et al. Sequential evaluation for bone union of transferred fibula flaps in reconstructed mandibles: panoramic X-ray versus computed tomography. Int J Oral Maxillofac Surg 2015; 44: 942-947. doi:10.1016/j. ijom.2015.04.014

[34] Moubayed SP, L'Heureux-Lebeau B, Christopoulos A et al. Osteocutaneous free flaps for mandibular reconstruction: systematic review of their frequency of use and a preliminary quality of life comparison. J Laryngol Otol 2014; 128: 10341043. doi: $10.1017 /$ S0022215114002278
[35] Lonie S, Herle P, Paddle A et al. Mandibular reconstruction: meta-analysis of iliac- versus fibula-free flaps. ANZ J Surg 2015; 86: 337-342. doi:10.1111/ans.13274

[36] Zosa BM, Elliott CW, Kurlander DE et al. Facing the facts on prophylactic antibiotics for facial fractures: 1 day or less. J Trauma Acute Care Surg 2018; 85: 444-450. doi:10.1097/ TA.0000000000002009

[37] Lalezari S, Lee C, Paydar KZ et al. Age and number of surgeries increase risk for complications in polytrauma patients with operative maxillofacial fractures. World J Plast Surg 2018; 7 : 307-313. doi:10.29252/wjps.7.3.307

[38] Grohmann I, Raith S, Mücke T et al. Biomechanical loading test on reconstructed mandibles with fibular, iliac crest or scapula graft: a comparative study. Br J Oral Maxillofac Surg 2015; 53: 741-747. doi:10.1016/j.bjoms.2015.05.022

[39] Shen Y, Guo X-H, Sun J et al. Double-barrel vascularised fibula graft in mandibular reconstruction: a 10-year experience with an algorithm. J Plast Reconstr Aesthet Surg 2013; 66: 364371. doi:10.1016/j.bjps.2012.10.005

[40] Bolzoni A, Mapelli A, Baj A et al. Evaluation of three-dimensional mandibular movements after reconstruction with free fibula flap. Acta Otorhinolaryngol Ital 2015; 35: 371-378. doi:10.14639/0392-100X-504

[41] Jewer DD, Boyd JB, Manktelow RT et al. Orofacial and mandibular reconstruction with the iliac crest free flap: a review of 60 cases and a new method of classification. Plast Reconstr Surg 1989; 84: 391-403

[42] Ferreira J], Zagalo CM, Oliveira ML et al. Mandible reconstruction: history, state of the art and persistent problems. Prosthet Orthot Int 2014; 39: 182-189. doi:10.1177/ 0309364613520032

[43] Kniha K, Kniha H, Grunert I et al. Esthetic Evaluation of Maxillary Single-Tooth Zirconia Implants in the Esthetic zone. Int J Periodontics Restorative Dent 2018. doi:10.11607/prd.3282

[44] Möhlhenrich SC, Kniha K, Elvers D et al. Intraosseous stability of dental implants in free revascularized fibula and iliac crest bone flaps. J Craniomaxillofac Surg 2016; 44: 1935-1939. doi:10.1016/j.jcms.2016.09.011

[45] Wijbenga JG, Schepers RH, Werker PMN et al. A systematic review of functional outcome and quality of life following reconstruction of maxillofacial defects using vascularized free fibula flaps and dental rehabilitation reveals poor data quality. J Plast Reconstr Aesthet Surg 2016; 69: 1024-1036. doi:10.1016/j. bjps.2016.05.003

Bibliografie

DOI https://doi.org/10.1055/a-0898-4690 OP-JOURNAL 2019; 35: 262-273 @ Georg Thieme Verlag KC Stuttgart · New York ISSN 0178-1715 